\title{
Die Anwendung des Zinks zur Reduktion bei der Bestimmung von Vanadinsäure.
}

\author{
Von
}

\author{
F. A. Gooch und R. D. GILBERt. ${ }^{1}$
}

Die Reduktion der Vanadinsäure zu Vanadintetroxyd für die titrimetrische Bestimmung mit Kaliumpermanganat wird gewöhnlich mit Schwefeldioxyd oder mit Schwefelwasserstoff ausgeführt. Die einfachere Behandlung mit Zink in saurer Lösung ist nicht direkt anwendbar, weil die Vanadinsäure unter diesen Verhältnissen in unregelmälsiger Weise annähernd bis zum Dioxyd reduziert wird, wie zuerst Czudnowicz ${ }^{2}$ gezeigt hat. Die im folgenden mitgeteilten Versuche hatten den Zweck, eine zuverlässige Methode ausfindig zu machen, um das durch Zink in saurer Lösung resultierende Reduktionsprodukt der Vanadinsäure mit Sicherheit in vierwertiges Vanadin überzuführen. Wenn dies möglich ist, so könnte das für die Vorbereitung von Eisensalzen zur Titration mit Kaliumpermanganat so wertvolle Reduktionsmittel zu dem gleichen Zwecke auch für die Bestimmung der Vanadinsäure in ihren Salzen Verwendung finden.

Die Anwendung von Zink zur Reduktion erfolgt am zweckmälsigsten in der folgenden Weise: ${ }^{8}$ Das eingezogene Ende eines Glasrohres von $2 \mathrm{~cm}$ innerem Durchmesser und $50 \mathrm{~cm}$ Länge wird an einen Hahn angeschmolzen, an dem andererseits ein Rohr von $0.5 \mathrm{~cm}$ innerem Durchmesser und $24 \mathrm{~cm}$ Länge sitzt. In die Verengung des weiteren Rohres wird ein Stück Platindrahtnetz gelegt und auf dieses eine etwa $2 \mathrm{~cm}$ starke Schicht von Glaswolle; diese

1 Ins Deutsche übertragen von J. Koppes.

2 Ann. Phys. 120 (1863), 39.

${ }^{3}$ Beschrieben in Blains Chemical Analysis of Iron (1902), S. 93. 
bedeckt man schliefslich mit einer ca. $40 \mathrm{~cm}$ hohen Säule von amalgamierten Zinkstücken, die so grofs sind, dafs sie durch ein Sieb mit 8 Maschen auf den Zentimeter hindurchgehen. Das engere Rohr geht durch einen Gummistopfen auf einem Vakuumkolben hindurch; der letztere steht durch einen Druckregulator mit einer Luftpumpe in Verbindung. Bei der Benutzung des Apparates wird zunächst die Pumpe in Gang gesetzt und der Regulator so eingestellt, dafs etwa ein Unterdruck von $20 \mathrm{~cm}$ Wasser entsteht. Hierauf wärmt man das Zink durch Einsaugen von heifsem destillierten Wasser und $100 \mathrm{ccm}$ heilser einprozentiger Schwefelsäure an und lärst dann die zu reduzierende Vanadinlösung in kleinen Portionen durch das Zink eintreten, abwechselnd mit kleinen Portionen einprozentiger Schwefelsäure (ca. $100 \mathrm{ccm}$ in ganzen). Schliefslich wäscht man das Zink noch mit etwa $100 \mathrm{ccm}$ der verdünnten Säure und etwa $250 \mathrm{ccm}$ destilliertem Wasser. Während der ganzen Operation mufs das Zink von Flüssigkeit bedeckt bleiben, so dafs es nicht mit der Luft in Berührung kommt.

Die lavendelblaue Flüssigkeit im Kolben enthält Vanadindioxyd; sie nimmt, wie Roscos ${ }^{1}$ gezeigt hat, beim Durchleiten eines Luftstromes die blaue Farbe der Vanadintetroxydlösungen an. Die ersten Versuche, einen gut definierten Oxydationszustand zu erreichen, wurden im Anschlufs an Roscoes Beobachtung gemacht. Wir behandelten die im Kolben gesammelte Flüssigkeit bei verschiedenen Temperaturen und bei wechselnder Zeitdauer mit Luft und titrierten sie sodann nach dem Erwärmen auf $80^{\circ}$ mit $1 / 20 n$ Kaliumpermanganatlösung, die gegen $1 /{ }_{20} n$-Arsentrioxydlösung eingestellt war.

Diese sehr genaue Methode zur Einstellung der Permanganatlösung führten wir derart aus, dafs eine passende Menge (43 ccm) der Permanganatlösung zu einer mit $3 \mathrm{ccm}$ Schwefelsäure $(1: 1)$ angesäuerten Jodkaliumlösung ( $3 \mathrm{~g} \mathrm{KJ}$ ) in einer mit Hahntrichter versehenen Flasche gegeben, in diese sodann titrierte $1 / 20 n$-Arsentrioxydlösung im Überschuls hineingelassen und nach dem Neutralisieren mit saurem Kaliumkarbonat unter Stärkezusatz mit Jod zurücktitriert wurde.

Bei Blindversuchen mit dem Reduktionsrohr wurde gefunden, dafs der Farbumschlag in der sauren Lösung schon nach Zusatz von $0.2 \mathrm{ccm}$ Kaliumpermanganat erfolgte; diese Korrektion ist be-

${ }^{1}$ Ann. Chem. Pharm., Suppl. 6 (1868), 98. 
dingt durch Spuren von Eisen in dem angewandten Zink und durch das grofse Volumen der Lösung; sie wurde bei allen Vanadinbestimmungen in Rechnung gezogen. Für unsere Versuche benutzten wir Ammoniummetavanadat, und zwar ein Präparat mit $76.66 \%$ $\mathrm{V}_{2} \mathrm{O}_{5}$, dessen Gehalt nach HoLversc日eits jodometrischer Methode festgestellt worden war. ${ }^{1}$

Die Resultate einiger Versuche, die Oxydation der reduzierten Lösungen zu Tetroxyd unter sehr günstigen Verhältnissen durch Luft zu bewirken, sind in Tabelle I zusammengestellt. Obgleich nun dem Anscheine nach die Oxydation durch Luft glatt bis zu Ende verläuft, so erkennt man doch aus den Zahlen, dals die völlige Umwandlung der niedrigen Oxyde in Tetroxyd nur langsam und in wenig sicherer Weise nach dem angegebenen Verfahren erfolgt, so dals dasselbe nicht als Basis einer zuverlässigen und schnellen quantitativen Methode dienen kann.

Tabelle I.

\begin{tabular}{c|c|c|c|c}
\hline $\begin{array}{c}\text { Zeitdauer der } \\
\text { Behandlung mit } \\
\text { Luft (Minuten) }\end{array}$ & $\begin{array}{c}\text { Temperatur } \\
\text { in } 0\end{array}$ & $\begin{array}{c}\text { Angew. } \\
\mathrm{V}_{2} \mathrm{O}_{5}\end{array}$ & $\begin{array}{c}\text { Gef. } \mathrm{V}_{2} \mathrm{O}_{5} \\
\left(\mathrm{mit} \mathrm{KMnO}_{4}\right)\end{array}$ & Fehler \\
\hline & $\mathrm{g}$ & $\mathrm{g}$ & $\mathrm{g}$ \\
\hline 75 & $56-28$ & 0.0767 & 0.0778 & +0.0011 \\
75 & $60-29$ & 0.0767 & 0.0805 & +0.0038 \\
75 & $48-29$ & 0.0767 & 0.0829 & +0.0062 \\
75 & $* 100$ & 0.0767 & 0.0772 & +0.0005 \\
75 & $* 100$ & 0.0767 & 0.0769 & +0.0002
\end{tabular}
erhitzt.

* Die Lösung wurde in dem angegebenen Intervall zweimal zum Kochen

Die Einwirkung des molekularen Luftsauerstoffs ist offenbar nicht hinreichend, um alle niedrigen Oxyde in saurer Lösung vollständig in nicht allzu langer Zeit in Vanadintetroxyd überzuführen; die üblichen Oxydationsmittel aber bewirken auch eine höhere Oxydation des Tetroxyds. Wir haben nun gefunden, dafs Silberoxyd und Silbersalze zwar die niedrigen Oxyde angreifen, das Tetroxyd jedoch unverändert lassen. Silbersulfat scheint das geeignetste Salz für die Oxydation der reduzierten Lösung zu sein.

Bei unseren Versuchen mit Silbersulfat wurde die Vanadinsäurelösung in dem Reduktionsrohr genau in der beschriebenen

1. Inaug.-Diss., Berlin 1890, S. 49. 
Weise behandelt; nur wurde vor Beginn des Versuches der Aufnahmekolben mit Silbersulfatlösung beschickt. Bei den ersten sechs Versuchen wandten wir $100 \mathrm{ccm}$, bei den zwei letzten je $300 \mathrm{ccm}$ gesättigte Silbersulfatlösung an. Der Kolbeninhalt wurde gekocht und dann durch Asbest in einem Filtriertiegel filtiert. Nach dem nochmaligen Erhitzen der Lösung (ca. $700 \mathrm{ccm}$ ) zum Sieden wurde mit $1 /{ }_{20} n$-Kaliumpermanganat titriert. Wenn die reduzierte Vanadinlösung aus dem Reduktionsrohr in die Silberlösung eintritt, so erfolgt Abscheidung von fein verteiltem metallischen Silber in Form von Schlamm; kocht man dann jedoch die Mischung auf, so vereinigt sich das ganze Silber zu einer schwammigen Masse, so dals man ohne Filtration titrieren könnte, wenn nicht schwammförmiges Silber von Permanganat leicht angegriffen würde. ${ }^{1}$

Tabelle II.

\begin{tabular}{c|c|c|c}
\hline $\begin{array}{c}\text { Angew. } \mathrm{V}_{2} \mathrm{O}_{5} \\
\mathrm{~g}\end{array}$ & $\begin{array}{c}\text { Verbraucht: } \\
\mathrm{KMnO}_{4} \text { in cem }\end{array}$ & $\begin{array}{c}\text { Gef. } \mathrm{V}_{2} \mathrm{O}_{5} \\
\mathrm{~g}\end{array}$ & $\begin{array}{c}\text { Fehler } \\
\mathrm{g}\end{array}$ \\
\hline 0.0767 & 17 & 0.0770 & +0.0003 \\
00767 & 17.04 & 0.0771 & +0.0004 \\
0.0767 & 17.05 & 0.0772 & +0.0005 \\
0.0767 & 16.96 & 0.0768 & +0.0001 \\
0.0767 & 16.98 & 0.0769 & +0.0002 \\
0.0767 & 17 & 0.0770 & +0.0003 \\
0.1918 & 42.9 & 0.1942 & +0.0024 \\
0.1918 & 42.7 & 0.1933 & +0.0015
\end{tabular}

Aus den hier zusammengestellten Ergebnissen ist zu erkennen, dafs die Methode für geringere Vanadinmengen übereinstimmende und zuverlässige Werte gibt. Die gröfseren Abweichungen bei den beiden letzten Versuchen sind wahrscheinlich dadurch veranlafst, dafs es schwierig ist, den Umschlag in Rot als Endreaktion zu erkennen, wenn die rötlich-gelbe Färbung vorhanden ist, die in Gegenwart beträchtlicher Vanadinsäuremengen auftritt. Diese Schwierigkeit ist mit der Titration von grölseren Mengen Vanadin durch Permanganat unlösbar verknüpft.

1 Giles, Chem. News 15 (1867), 204; Otto von Der Prordten, Ber. deutsch. chem. Ges. 20, 3375; Friedheim, Ber. deutsch. chem. Ges. 20, 2554.

The Kent Chemical Laboratory of Yale University, New Haven, U. S. A.

Bei der Redaktion eingegangen am 13. April 1903. 